\title{
Sustainable production of graphene from petroleum coke using electrochemical exfoliation
}

\author{
Sanjit Saha $\mathbb{D}^{1}$, Pritishma Lakhe ${ }^{1}$, Matthew J. Mason ${ }^{1}$, Bryan J. Coleman ${ }^{1}$, Kailash Arole $\mathbb{I}^{1}$, Xiaofei Zhao ${ }^{1}$, Sergey Yakovlev ${ }^{2}$, \\ Sundararajan Uppili' ${ }^{2}$, Micah J. Green $\left(\mathbb{D}^{1 凶}\right.$ and Rohan A. Hule $\left(\mathbb{D}^{2 凶}\right.$
}

Petroleum coke is a solid, carbonaceous by-product of oil refining and is normally used for heating or as an anode in aluminum and steel production. These applications contribute to carbon emissions, but here we show that petroleum coke has another potential avenue: as a precursor for graphene production. This path presents an environmentally and economically sustainable use for a lowvalue industrial stream. Electrochemical exfoliation is used to produce graphene nanosheets from petroleum coke, rather than graphite. The final product is separated from the unreacted material by a two-step centrifuging process. SEM and TEM images confirm that the final product contains few-layered nanosheets, and the Raman spectra confirm that the exfoliated coke product is indeed graphene. Post-annealing of this product substantially increases the electrical conductivity. This new finding holds potential for the petroleum industry to produce a value-added nanomaterial and enhance the economic impact of slurry oil and slurry oilderived coke streams by orders of magnitude; this route also allows these streams to be directed away from high-emissions uses.

npj 2D Materials and Applications (2021)5:75; https://doi.org/10.1038/s41699-021-00255-8

\section{INTRODUCTION}

With the continuing rise in concern over sustainable resource use, the petrochemical industry faces challenges in managing each of its product streams. Even by-products of oil refining, such as petroleum coke, are difficult to utilize sustainably; coke is produced by heating resids or slurry oils from refinery units such as Fluid Catalytic Crackers. Coke is used as a fuel for heating in several industries, and the combustion of coke produces more $\mathrm{CO}_{2}$ per fuel mass than coal ${ }^{1}$. Coke is also used in the steel and aluminum industries as an anode for smelting, a process that also emits greenhouse gases ${ }^{2}$. These concerns highlight the global need to repurpose existing petroleum streams such as coke and its precursor oils toward sustainable end-uses (Fig. 1).

Here, we demonstrate the use of petroleum coke as a feedstock for carbon nanomaterial production. Graphene, in particular, is an exciting target because of its ongoing deployment into a range of application fields including batteries, supercapacitors, structural materials, transparent electronics, and flexible wearable devices ${ }^{3,4}$. It is highly desirable to expand the suite of graphene precursors to include existing industrial by-products.

The economics of possible coke-to-graphene processes are quite striking: needle coke, the highest quality grade of petroleum coke, can be used for electrodes in steel production and can be purchased for $\sim$ 1500-3000/ton. Bulk graphene powder can be purchased at the lab scale for as little as $\sim \$ 8 /$ gram, orders of magnitude more valuable than coke. This latter price will decrease with scaleup, but this is still an order-of-magnitude increase in economic value.

In addition, petroleum coke provides an additional feedstock for graphene production. Natural graphite is a finite source; it is estimated that 800 million tons can be recovered worldwide 5 . Furthermore, much of it is difficult to use or unusable for graphene production because only $10-15 \%$ of natural graphite is actually graphitic carbon; most of it is amorphous and contains silicate minerals or metals ${ }^{5}$. In contrast, needle coke can be consistently produced with high graphitic content and low impurity concentrations. Global needle coke production was at 1.1 million tons per year as of 2020, and it is expected to increase to 1.5 million tons per year by $2026^{6}$. However, these numbers are based on the demand for needle coke for the steel and lithium-ion battery industries; needle coke production can be significantly increased to meet additional demand if needed. Although petroleum (and therefore petroleum coke) is also a finite resource, progress has been made toward producing needle coke from renewable feedstocks such as biomass ${ }^{7}$ or plastic waste ${ }^{8}$. Not only can needle coke be a more permanent feedstock for graphene production, but this avenue also the petroleum portfolio away from high-emission end-uses.

Graphite-derived graphene is well-documented ${ }^{9-11}$, but cokederived graphene has not been extensively explored. Prior work on the production of graphene from coke has largely focused on graphene oxide (GO) and explored the effect of crystallinity on the resulting lateral size ${ }^{10,12,13}$. Ball-milling coke with stearic acid has also been explored ${ }^{14}$, but questions remain about the distinction between the parent material and the final graphene-like product, particularly in their Raman signature. This is likely related to a lack of effective separation procedures in place.

Our group recently demonstrated the potential for high-yield and scalable production of high-quality graphene nanosheets using an electrochemical exfoliation (ECE) method in a compressed expandable chamber ${ }^{15,16}$. The compressed reactor allows for expansion of the parent material as it is exfoliated while maintaining electrical contact throughout the mixture. (This is superior to conventional electrochemical methods that use graphite monoliths as a parent material; the exfoliation process breaks apart the graphite monolith, losing electrical contact and halting the reaction.) The resulting nanosheets are termed electrochemically exfoliated graphene (EEG). Simpler ECE procedures have been attempted on coke before, but the resulting 


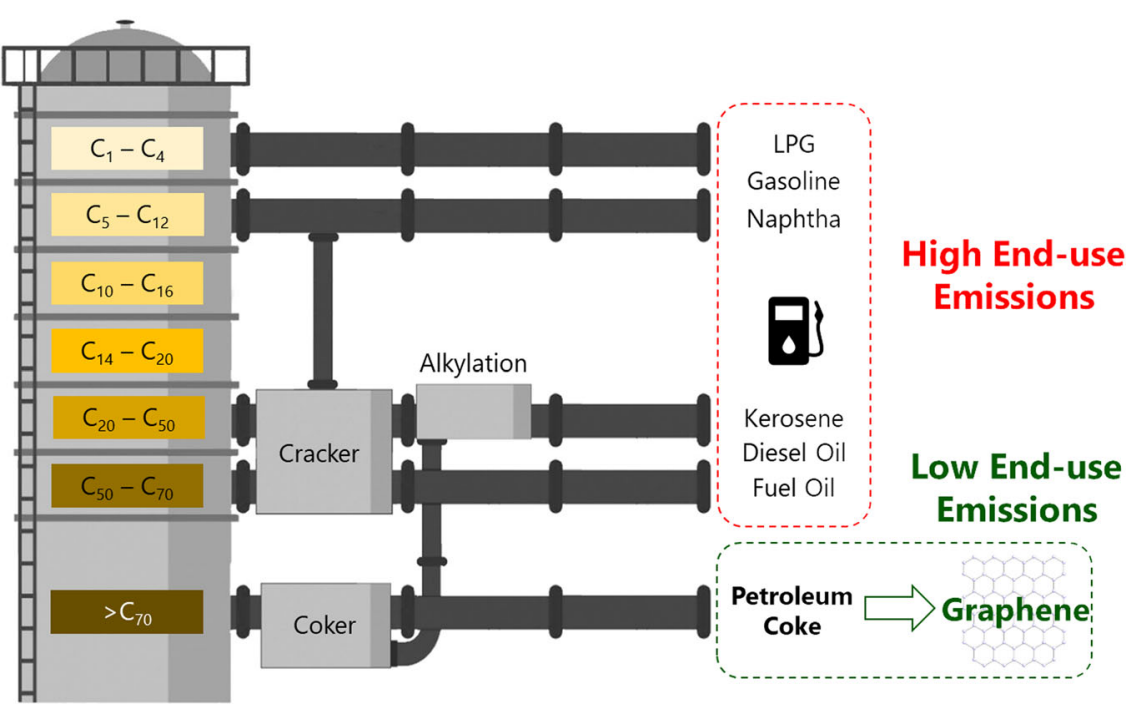

Fig. 1 Schematic of Refinery Products. Simplified schematic of refinery operations and products: there is a need to push these petroleum streams toward products with low end-use emissions, such as carbon nanomaterials.

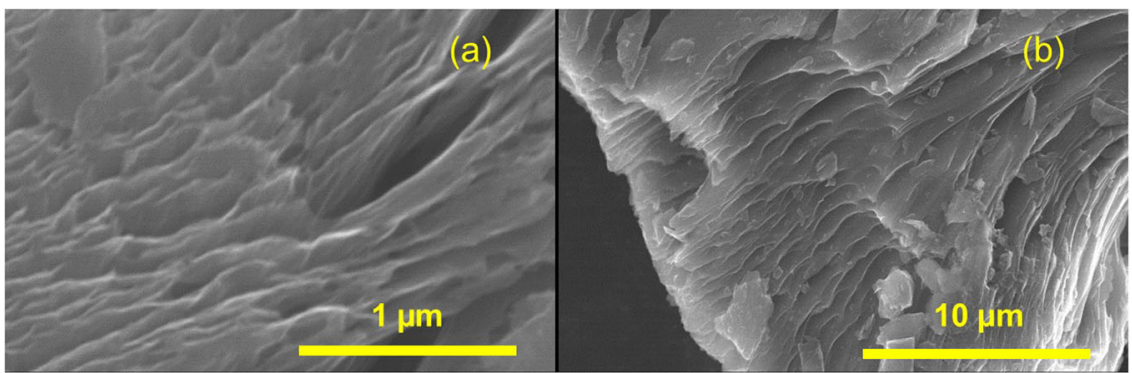

Fig. 2 FE-SEM image of CK-1 parent material. The sample contains both (a) small and (b) relatively large $(30-100 \mu \mathrm{m})$ particles.

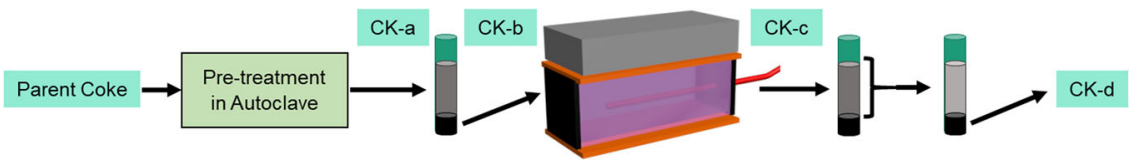

Fig. 3 Schematic of the coke-graphene synthesis process. It contains four steps as described in the scheme as follows: pre-treatment, prereaction separation, electrochemical exfoliation process, post-reaction separation process.

material either formed small graphene quantum dots or required additional post-processing exfoliation ${ }^{17,18}$.

In this work, different needle coke grades are evaluated as the parent material for the ECE process in a compressed chamber. The reactor product contained unreacted material, unwanted small particles, and a graphene-like product, termed Coke-EEG, which was isolated by a two-step centrifugation method. The morphology and composition of the final product confirm that this material is indeed a graphene-family material. This finding is at the core of a new industrial push toward the use of natural gas and petroleum streams to produce high-value nanomaterials ${ }^{19-23}$.

\section{RESULTS AND DISCUSSION \\ ECE of coke}

Here, we show that petroleum cokes can be electrochemically exfoliated into graphene-like structures. The ECE method depends on electrolyte intercalating between the layers of precursor material to achieve the best results. Thus, a more layered, crystalline coke precursor has more potential for graphene production. Needle coke is highly crystalline and has long needle-like structures. The high aromaticity and layered structure make needle coke the most promising coke candidate to act as a graphene precursor. The field emission scanning electron microscope (FE-SEM) images in Fig. 2 show the layered structure of CK1 , with layer thicknesses that can be $<100 \mathrm{~nm}$. (More images corresponding to this and other cokes are presented in Supplementary Figs. 1-3)

Figure 3 represents the ECE process, in which the starting material is the parent coke, and the final product is then exfoliated and separated coke (CK-d). The ECE process contains four steps described in detail in Supplementary Fig. 4. Supplementary Fig. 4a describes the pre-treatment and washing process. The nitric acid pre-treatment allows for a more hydrophilic (and wettable) starting material, allowing for better exposure to the electrolyte solution during the ECE process. Supplementary Fig. 4b shows the pre-reaction separation process through centrifugation at $4000 \mathrm{rpm}$ for $10 \mathrm{~min}$. The separated parent material (sediment) was used as the starting material for the ECE process (Supplementary Fig. 4c), which was carried out at $12 \mathrm{~V}$ for $2 \mathrm{~h}$. This voltage is unusually high relative to that used for graphite ECE. However, below this voltage, the yield of coke-EEG is quite low. Above $12 \mathrm{~V}$, the membrane itself may be damaged. The CK-b samples are compacted inside a hollow cellulose membrane. To keep the working electrode (CK-b) compacted into an electrically 
conducting monolith, an external weight is placed on top of the membrane. Both the working and counter electrodes are submerged in the electrolyte. Under a positive bias at the working electrode, CK-b samples are then exfoliated to CK-c (EEG). After the reaction, the reaction mixture is washed and then suspended in an ethanol-water mixture. The ECE product contained nonexfoliated coke, smaller non-graphitic material, and the EEG product. To separate the graphene sheets (final product) from the undesired material, a two-step centrifuge process was followed as described in Supplementary Fig. 4d; the first centrifuge removes the unreacted parent material, and the second centrifuge removes the small, non-graphitic material.

\section{Characterization of coke-derived graphene}

The FE-SEM images of CK-1b and CK-1c, the parent material and product of the ECE process, are shown in Supplementary Fig. 5a and Supplementary Fig. 5b, respectively. These images show that the ECE process breaks down some of the $40 \mu \mathrm{m}$-sized parent material particles into smaller sheet-like graphene platelets and some smaller non-graphitic particles.

More importantly, graphene-like nanosheets are clearly seen in FESEM images (Fig. 4a, b) of CK-1d (final product, reacted and then separated), confirming the major hypothesis of the paper. The lateral dimension of the coke-graphene sheets varies considerably, with the majority of lateral sizes in the $1-3 \mu \mathrm{m}$ range. The presence of graphene sheets was further investigated by TEM imaging. Figure 4c, d show HR-TEM images of CK-1d where graphene-like sheets are clearly visible (additional TEM images can be found in Supplementary Figs. 6, 7). Moreover, high-resolution imaging reveals a hexagonally packed crystalline lattice of sheets as seen in Fig. 4d. This lattice corresponds to a graphitic structure and is virtually identical to the structure observed on graphite-derived EEG shown in Supplementary Fig. 8. The sheets appear to be a single crystal, with no grain boundaries observed in the micrograph field of view. (However, sheets frequently contain folds that may potentially mask such features.) The coke-derived product has a smaller fraction of very large particles relative to graphite-derived EEG, likely owing to the thorough post-processing separation.

The yield of the coke-derived graphene product was $~ 1.6 \%$, which is lower than typical EEG yields of $10 \%$ or more (depending on reaction conditions) reported in our prior work, indicating that coke is more difficult to exfoliate than graphite ${ }^{15,16}$. However, this value is far lower than the true yield because of the need for the centrifuge process post reaction to isolate EEG from both parent material and impurities. Our prior work indicates that significant graphene product remains in the unwanted material and can be recovered via multiple separation passes ${ }^{24}$. The remaining material may also be recycled back through the process as a parent material with no pre-treatment.

In contrast, the ECE products of CK-2 and CK-3 did not contain a significant amount of well-defined, graphene-like sheets. Supplementary Fig. 9a shows an FE-SEM image of CK-3d. Although a few large sheets are visible, most of the structures are not graphenelike but are more similar to large needle-like structures. In addition, Supplementary Fig. 9b shows an FE-SEM image of CK-2d; graphene-like sheets are not visible in this image either. The presence of large plate-like and needle-like structures suggests that graphene was not successfully prepared from CK-2 or CK-3 by the ECE process. TEM images of CK-3d (Supplementary Fig. 9c) and CK-2d (Supplementary Fig. 9d) also do not show any sheet-like structures. These ECE products (CK-2d, CK-3d) are quite different than the graphene-like structure in $\mathrm{CK}-1 \mathrm{~d}$. Potential reasons for this difference may relate to the parent material structure.

Petroleum cokes are produced in a variety of manners, many of which are proprietary. Although specific production details of each coke are unknown, optical microscopy was carried out on each parent material to distinguish their morphology. Note that CK-2 and CK-3 were received as relatively large pieces and required

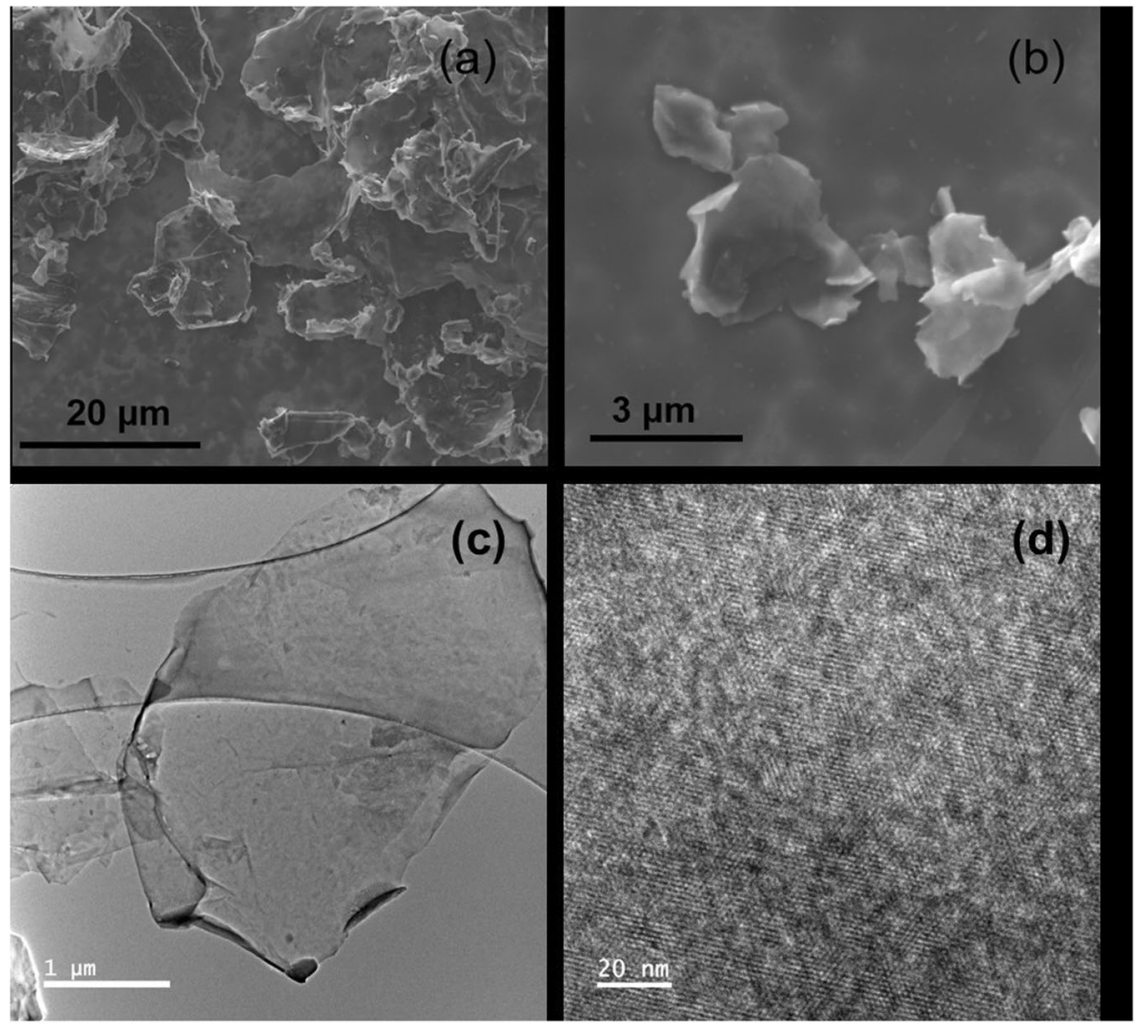

Fig. 4 Electron Microscopy Analysis. Scanning electron microscopy: a and b SEM images of CK-1d (EEG, separated) and c and d TEM images of CK-1d. 


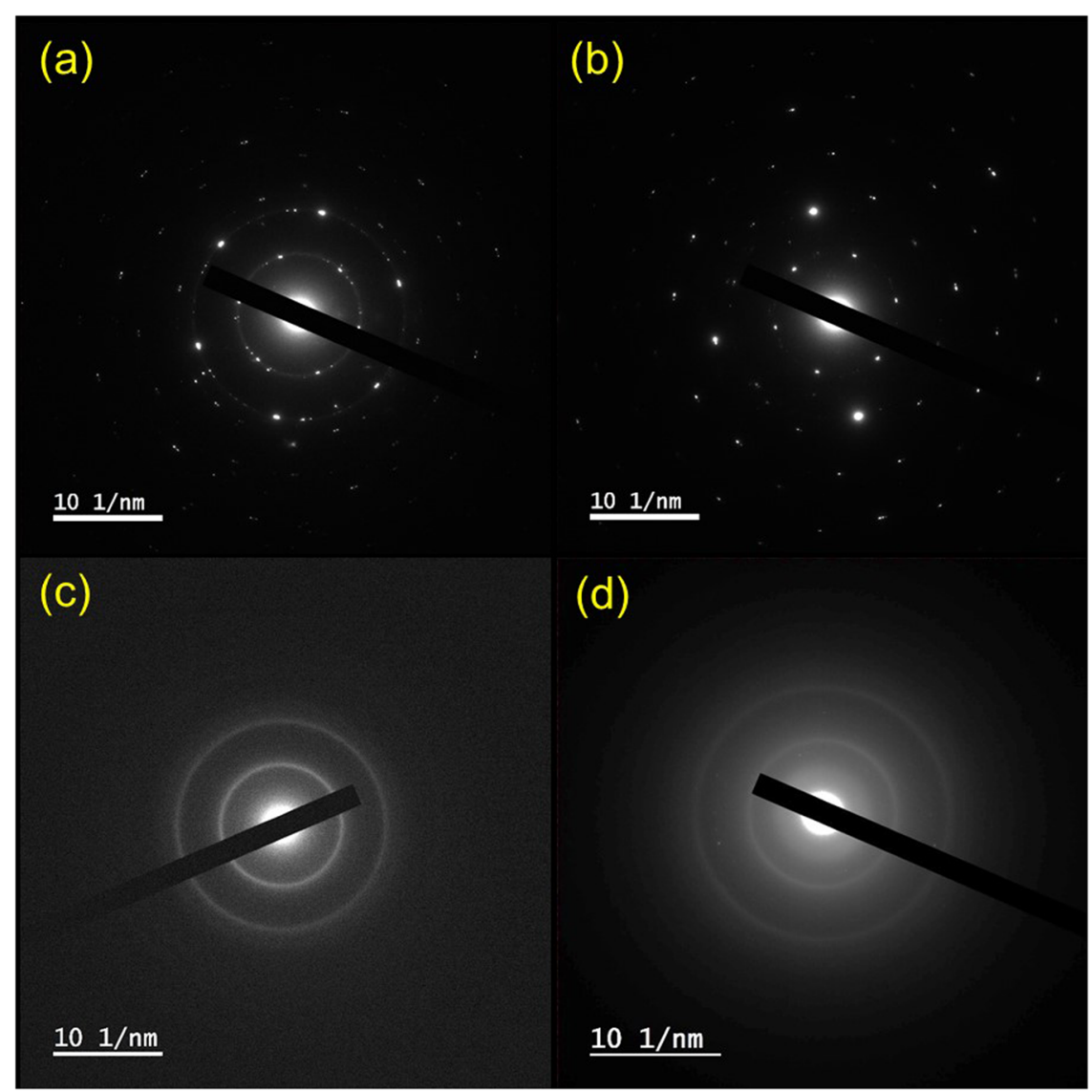

Fig. 5 Diffraction Patterns of Coke and Coke-derived graphene. Electron diffraction patterns of a CK-1d EEG, b graphite-derived EEG, c CK2d EEG, and d CK-1 coke.

grinding prior to the ECE process. The CK-1 powder was very fine and has a significantly smaller particle size than CK-2 and CK-3. The CK-1 particle edges are more jagged, angular, and distorted, whereas the CK-2 and CK-3 particles have more rounded edges and no layers. These morphology differences may allow for differences in acid effects, electrolyte intercalation, and ease in layer exfoliation. Optical images of each parent material are included in Supplementary Figs. 10-12. Aside from these morphology factors, the three-parent needle cokes are quite similar, including in their Raman and TGA data (discussed below).

The electron diffraction pattern of CK-1d (Fig. 5a) shows a crystalline structure with a well-ordered hexagonal arrangement of atoms, confirming the formation of graphene-like nanosheets. This pattern observed on CK-1d is similar to patterns observed in graphite-derived EEG (Fig. 5b). In contrast, the electron diffraction pattern of the CK-1 coke parent material (Fig. 5c) shows broad (amorphous) rings. Similarly, CK-2d (Fig. 5d) shows similar features, indicating an amorphous final product. There is a signature of these same features in the pattern for CK-1d, suggesting higher amorphous content than graphite-derived EEG.

The oxidation content in the parent coke and EEG product can be assessed in multiple ways: in our prior work, we showed that thermogravimetric analysis (TGA) can be used as a rough measure of oxygen content. TGA analysis of the parent coke and EEG product is shown in Fig. 6a. At $\sim 700^{\circ} \mathrm{C}$, half of CK-1d is removed ${ }^{15}$. At the same temperature $\left(700^{\circ} \mathrm{C}\right)$, only $\sim 10 \%$ of the mass was removed for $\mathrm{CK}-1 \mathrm{a}$, indicating that the degree of oxidation is higher for CK-1d than CK$1 \mathrm{a}$; this indicates that some oxidation may occur during ECE. However, the degree of oxidation is lower than that seen in GO or even in graphite-derived EEG ${ }^{15}$. X-ray photoelectron spectroscopy (XPS) was used to determine the surface composition of the CK-1d sample shown in Supplementary Fig. S13. CK-1d material has oxygen to carbon atomic ratio $(\mathrm{O}: \mathrm{C})$ of 0.29 which remains relatively low; in addition, we did not observe much $\mathrm{C}-\mathrm{O}$ bonding based on the $\mathrm{C} 1 \mathrm{~s}$ peak fittings. This suggests a product that is more similar to graphene than $\mathrm{GO}^{25}$. Nitrogen element was not observed for this sample, indicated by the XPS survey spectra (no peak shows $399 \mathrm{eV}$ where $\mathrm{N} 1 \mathrm{~s}$ peak should be).

Electron energy loss analysis was also performed to evaluate the level of oxidation. It was shown in prior work that GO has a sharp peak immediately after the $\pi^{*}$ excitation peak at $\sim 279 \mathrm{eV}$ in carbon k-edge $\mathrm{e}^{26}$. The magnitude of this peak increases with an increase in oxygen content. At $\sim 10-15 \%$ oxygen, the magnitude of this peak becomes equal to the magnitude of the $\pi^{*}$ peak. Figure $6 \mathrm{~b}$ shows the spectra collected from CK-1a, graphite, and EEG derived from graphite and the coke. Neither coke nor graphite shows the peak corresponding to oxidation. Graphitederived EEG contains $10-15 \%$ of oxygen, which is consistent with prior findings, whereas the coke-derived EEG is considerably less.

Raman spectra provide valuable information about carbonaceous materials. Generally, Raman generates two distinct peaks at $\sim 1350$ and $1570 \mathrm{~cm}^{-1}$ called the $D$ and $G$ band, respectively. The $\mathrm{G}$ band is related to the graphitic structure $\left(\mathrm{sp}^{2}\right)$, whereas the $D$ band indicates a distortion or defect $\left(\mathrm{sp}^{3}\right)$. In our data (Fig. $6 \mathrm{c}$ ), the relative intensity of the $\mathrm{G}$ band is significantly higher for CK-1d than for the CK-1 parent or CK-1c. This indicates the isolation of an $\mathrm{sp}^{2}$ structure. The Raman peak fitting results are displayed in Supplementary Fig. 14. In addition, more accurate values of the $I_{D} /$ $\mathrm{I}_{\mathrm{G}}$ ratios based on the intensities of the fitted $D$ and $G$ peaks are also included in Supplementary Fig. 14.

However, the most exciting finding was the appearance of a 2D peak in the CK-1d Raman spectra (Fig. 6c). The parent coke has no 

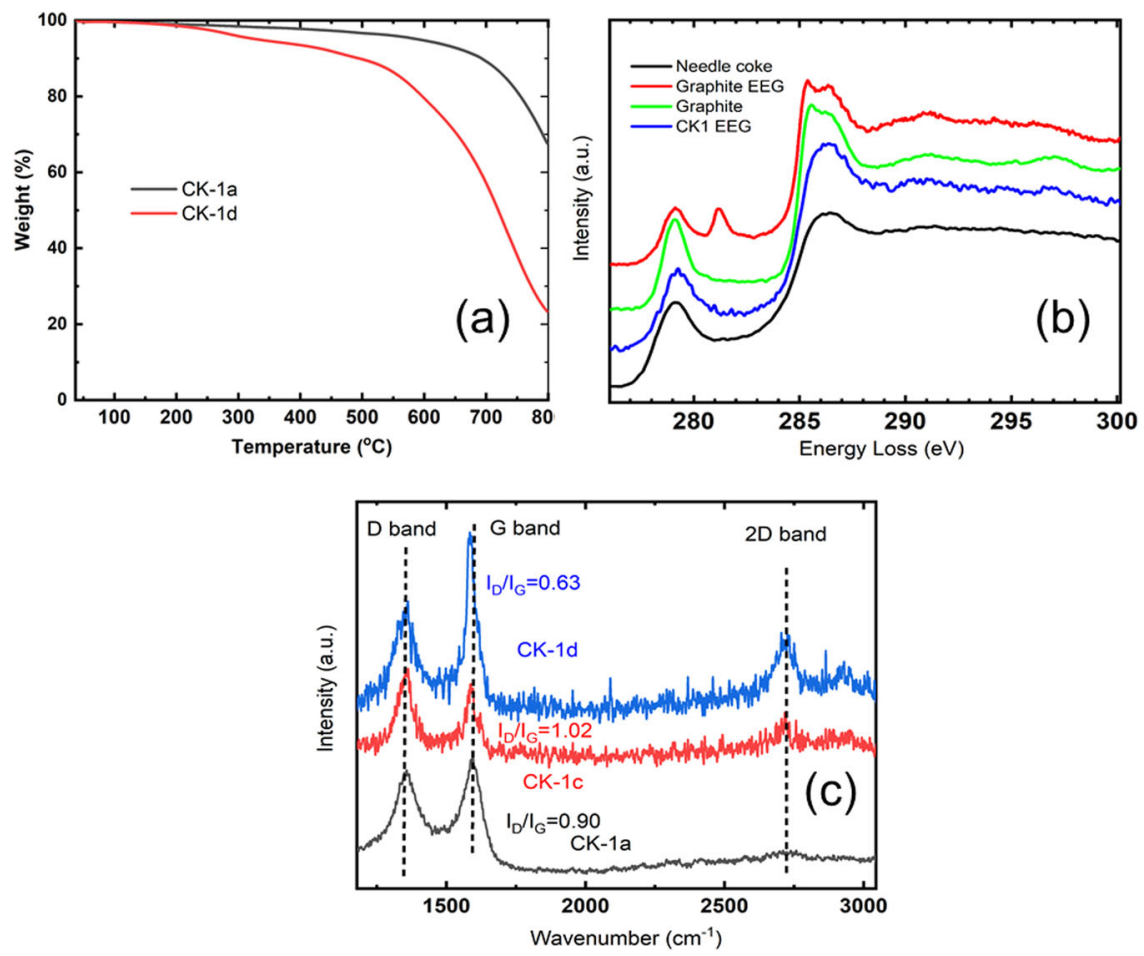

Fig. 6 Thermogravimetric and Raman Analysis. Thermogravimetric analysis: a TGA of CK-1a and CK-1d (inert atmosphere). TGA of CK-1d was carried out on freeze-dried material, b EELS spectra showing carbon k-edge in CK-1a, graphite, CK-1d EEG, and Graphite EEG, and c Raman spectra of CK-1a, CK-1C, and CK-1d show different ID/IG ratios and 2D band characteristics for the parent material, material after the ECE reaction, and after the post-reaction centrifuging process.

2D peak; this suggests that the parent coke lacks sufficient graphitic content to show this classic graphite signature. A small 2D hump is present in CK-1C, which indicates that after the ECE process, a significant amount of graphene-like material is present. The 2D peak is even larger (with no shoulder in the peak) in CK-1d, confirming that the centrifugation process separates the graphene product from unwanted material. Note that the difference between these 2D peaks indicates that our process is isolating the exfoliated graphene-like material from the parent material, not converting it; the lack of a $2 \mathrm{D}$ peak in the parent coke indicates that the maximum possible graphene yield will be considerably lower than that of graphite.

In contrast, no significant difference was observed in the Raman spectra for CK-2 and CK-3 before and after exfoliation. Supplementary Fig. 15 shows the Raman spectra of CK-2a, CK-2c, and CK$2 d$. The $I_{D} / I_{G}$ ratio of CK-2d was only slightly lower than CK-2a and CK-2c. However, this does suggest that the ECE process is still isolating primarily $\mathrm{sp}^{2}$ structures even from less crystalline precursors. A similar scenario is found for the Raman spectra of CK-3a, CK-3C, and CK-3d (Supplementary Fig. 16). XRD of the parent cokes (Supplementary Fig. 17) shows that all the starting material has (002) peaks at $26^{\circ}$.

Electrical properties and annealing of coke-derived graphene Vacuum-filtered films were made from the dispersions of both coke and coke-derived graphene. The electrical conductivities of parent CK-1, CK-2, and CK-3 were measured as 0.07, 0.23, and $0.38 \mathrm{~S} / \mathrm{m}$, respectively (Supplementary Table 1). Electrical conductivity increased significantly after the exfoliation and separation process; the electrical conductivities of the three CK-d samples are summarized in Table 1 . The electrical conductivity of CK-1d is high $(\sim 56.9 \mathrm{~S} / \mathrm{m})$, which can be attributed to its graphene-like structure. However, the other samples show relatively poor electrical conductivity. This is consistent with the
Table 1. Electrical conductivity of coke-derived graphene buckypapers, as well as a CK-1d sample annealed for $12 \mathrm{~h}$ at $1100^{\circ} \mathrm{C}$.

\begin{tabular}{llc}
\hline & Electrical conductivity $(\mathrm{S} / \mathrm{m})$ & Standard deviation \\
\hline CK-1d & 56.9 & 1.2 \\
CK-2d & 2.26 & 0.06 \\
CK-3d & 2.87 & 0.04 \\
CK-1d annealed & 474 & 11.9 \\
\hline
\end{tabular}

microscopy results described earlier: CK-1d is graphene-like, whereas CK-2d and CK-3d do not show a graphene-like morphology.

The electrical conductivity of the coke-derived graphene was lower than graphene-produced from graphite, so thermal annealing $\left(500^{\circ} \mathrm{C}\right.$ for $12 \mathrm{~h}$ in a tube furnace) was carried out on CK-1d to increase its electrical conductivity (Supplementary Table 2). This step significantly improved the electrical conductivities of both graphite-derived EEG and CK-1d (Supplementary Table 3) to 9735 and $250 \mathrm{~S} / \mathrm{m}$, respectively.

Additional thermal reduction and annealing were carried out at a range of temperatures and times for CK-1d to determine how both graphene structure and conductivity may be improved. These studies show that temperature has a much greater impact on electrical conductivity than time does (Table 2). When the annealing time was increased from 12 to $24 \mathrm{~h}$, the conductivity increased only slightly from 250 to $258 \mathrm{~S} / \mathrm{m}$ (at $500^{\circ} \mathrm{C}$ ). However, when the annealing temperature was increased from 500 to $900^{\circ} \mathrm{C}$, the electrical conductivity increased significantly from 250 to $345 \mathrm{~S} / \mathrm{m}$ (for $12 \mathrm{~h}$ of annealing). Furthermore, for annealing at $1100{ }^{\circ} \mathrm{C}$, the electrical conductivity is $474 \mathrm{~S} / \mathrm{m}$. For Li-ion battery applications, the required conductivity is between 10 and $1000 \mathrm{~S} /$ $\mathrm{m}$, so this is a suitable target for annealed CK- $1 d^{27,28}$. 
Table 2. Electrical conductivities of CK-1d after annealing at various times and temperatures.

\begin{tabular}{|c|c|c|c|c|c|c|}
\hline & CK-1d & $\begin{array}{l}\text { CK-1d } \mathrm{A} 1 \\
12 \mathrm{~h} \text { at } 500{ }^{\circ} \mathrm{C}\end{array}$ & $\begin{array}{l}\text { CK-1d A2 } \\
24 \mathrm{~h} \text { at } 500{ }^{\circ} \mathrm{C}\end{array}$ & $\begin{array}{l}\mathrm{CK}-1 \mathrm{~d} \mathrm{~A} 3 \\
12 \mathrm{~h} \text { at } 900{ }^{\circ} \mathrm{C}\end{array}$ & $\begin{array}{l}\mathrm{CK}-1 \mathrm{~d} \mathrm{~A} 4 \\
12 \mathrm{~h} \text { at } 1100^{\circ} \mathrm{C}\end{array}$ & $\begin{array}{l}\text { Graphite powder } \\
\text { (for Li-lon battery anode) })^{31}\end{array}$ \\
\hline Electrical conductivity (S/m) & $\sim 56.9$ & $\sim 250$ & $\sim 258$ & $\sim 345$ & $\sim 474$ & $\sim 166$ \\
\hline
\end{tabular}

Energy loss analysis of coke EEG annealed at various temperatures did not reveal any difference in electronic structure (Supplementary Fig. 18). One possible reason for the conductivity increase is the removal of covalently bound oxygen groups or adsorbed impurities. However, this should be achieved at $500^{\circ} \mathrm{C}$ and $12 \mathrm{~h}$. Beyond $500^{\circ} \mathrm{C}$, the conductivity further increases, possibly due to the removal of other impurities or molecular restructuring ${ }^{29,30}$. The relationship between temperature and conductivity is related to the restructuring process, but this process is not yet fully understood. Note that the improved conductivity may also result from improved graphenegraphene contacts in addition to the graphene structure changing during annealing. Considering the low initial content of oxygen in coke-derived EEG, we conclude that reduction is not the main reason for the conductivity increase. (Additional data on the TGA of these samples is found in Supporting Information, Supplementary Fig. 19).

\section{DISCUSSION}

In this work, needle cokes were explored as an alternative feedstock to produce graphene. Both microscopy and Raman indicate that graphene was successfully prepared from needle coke fines via the ECE process, with Raman analysis confirming that CK-1d contains few-layer graphene sheets. In this process, the centrifugation before the ECE reaction is important for refining the reaction feedstock, and the centrifugation after the reaction is crucial for separating the graphene product from the unreacted parent material. The final graphene material can be modified through annealing to achieve excellent conductivities appropriate for energy storage devices. These results show there is a promising pathway through which industry can sustainably use a by-product stream to produce a valuable low-emissions material. This also means that slurry oil (the precursor to many cokes) can be re-routed to produce coke and coke-derived graphene rather than high-emissions fuels. The emissions associated with the actual ECE process will decrease per mass if the process is scaled up. Furthermore, this process is economically advantageous, converting a low-value stream into a high-value nanomaterial product. High-value carbonates are a substantial market, depending on conductivity and aspect ratio, with values in the range of $\$ 10,000 /$ ton; production of coke-derived EEG feeds into both the composites and electronics sectors of this market.

\section{METHODS}

\section{Materials}

In the coking process, the heaviest components of petroleum are pressurized with steam and water to form solid petroleum coke. This process takes place inside a large vessel called a "coker unit". The raw coke that comes directly out of a coker unit is termed "green coke" and has $10-20 \mathrm{wt} \%$ volatile hydrocarbons. Petroleum cokes are categorized based on their application, with three major grades: fuel coke, anode coke, and needle coke ${ }^{31,32}$. A coker unit could produce one of these three grades depending on the process temperature, duration of coking, and the quality of hydrocarbon feedstocks. Low-grade vacuum residues produce the lowest value fuel coke as a by-product which is high in impurities (heavy metals, sulfur, and nitrogen) and is used as fuel in electricity generation and cement kilns. Relatively higher-grade vacuum residues are used to make anode grade coke, which has moderate value and is used in the aluminum industry. The highest quality and value, needle coke, is produced by coking high-quality aromatic feeds like Fluid Catalytic Cracker decant oil and is used to produce high-grade graphite electrodes for the steel industry ${ }^{29}$. These three cokes differ greatly in their microscopic and macroscopic structures. Anode coke has a sponge or honeycomb-like morphology, and the least crystalline fuel coke is made of agglomerates of spherical "shots". All of these cokes have no long order in their structure. Needle coke, on the other hand, is highly crystalline and has long needlelike structures. The high aromaticity and the ordered structure make needle coke the most promising candidate to act as a graphene precursor. The long-range order and aromaticity of needle coke can be further enhanced by heat treatment ${ }^{29}$. Needle coke can also be graphitized by heating in an inert atmosphere at $>2500^{\circ} \mathrm{C}$.

For this experimental work, dichloromethane and ammonium sulfate were purchased from Sigma-Aldrich. Ethanol was purchased from Fisher Chemical. Three different petroleum cokes (pet-cokes) were used as parent materials for the ECE process:

(i) Needle coke fines (CK-1),

(ii) Needle coke large particles (CK-2),

(iii) Needle coke medium size particles (CK-3)

The coke samples are modified as they go through the ECE process. To designate the material and stage of the process, a naming system is used as follows:

(a) Material that has been washed and pre-treated,

(b) Material that has been separated (before reaction),

(c) Material that has been electrochemically exfoliated (EEG),

(d) Material (EEG) that has been centrifuged (after reaction) and separated from the other reaction products. For example, needle coke (CK-2) that is between the separation and reaction steps is designated CK-2b.

\section{Experimental procedure}

Wash and pre-treatment process: the parent material was washed with dichloromethane and then deionized (DI) water via vacuum filtration to remove any impurities. The washed material was then pre-treated with $6 \mathrm{M}$ nitric acid by mixing it with the material, using $1 \mathrm{~mL}$ of acid per gram of material. The mixture was kept in a stainless-steel autoclave container and placed inside an oven at $120^{\circ} \mathrm{C}$ for $4 \mathrm{~h}$. The pre-treated material was washed with DI water to neutralize the acid then dried overnight at $100^{\circ} \mathrm{C}$ on a hotplate. The three washed and pre-treated cokes are designated as CK-1a, CK-2a, and CK-3a.

Pre-reaction separation process: the particles in each parent material varied greatly in size. Small particles, less fit for the reaction, were removed by centrifuging. The pre-treated material was mixed with water in a ratio of $5 \mathrm{mg} / \mathrm{mL}$ and centrifuged for $10 \mathrm{~min}$ at $4000 \mathrm{rpm}$. The supernatant containing the unwanted smaller particles was discarded, and the sediment was collected. The separated parent cokes are designated as CK-1b, CK-2b, and CK-3b. These CK-b materials were used as the starting material for the ECE process.

ECE setup and reaction process: the pre-treated and separated material was compacted in a dialysis bag (length $\sim 16 \mathrm{~cm}$ and width $\sim 2.5 \mathrm{~cm}$ ) that was clipped on both ends. A platinum wire was inserted into the material as the working electrode and copper mesh was wrapped around the dialysis bag as the counter electrode. A known weight $(\sim 0.75 \mathrm{~kg})$ was placed on top of the dialysis bag and copper mesh, and the apparatus was submerged in $2 \mathrm{~L}$ of $0.1 \mathrm{M}$ ammonium sulfate $\left((\mathrm{NH} 4)_{2} \mathrm{SO}_{4}\right)$ solution. A potential of $12 \mathrm{~V}$ was applied across the platinum electrode and copper mesh to power the ECE process, and the reaction was run for $2 \mathrm{~h}$. After reacting, the product was washed in DI water to remove the electrolyte. The ECE product contained unreacted materials, smaller non-graphitic material, and the EEG product. The unseparated reaction products are designated as CK-1c, CK-2c, and CK-3c.

Post-reaction centrifuging process: the post-reaction separation of the final product was carried out in a two-step centrifugation method. First, the 
unseparated material was centrifuged for $10 \mathrm{~min}$ at low speed (2000 rpm). In this step, unreacted larger particles were sedimented and discarded, and the supernatant was collected. Second, the supernatant was centrifuged again for $20 \mathrm{~min}$ at high speed $(5000 \mathrm{rpm})$. In this step, unreacted, nongraphitic smaller particles were removed in the supernatant. The sediment of the second step was collected as the final product; these separated coke-EEGs are designated as CK-1d, CK-2d, and CK-3d.

Yield calculation process: the yield of the coke-derived graphene (CK-1d) was measured from UV-vis absorbance spectroscopy using an extinction coefficient of $1293 \mathrm{~L} / \mathrm{g} / \mathrm{m}^{33}$. The absorbance measurement at $666 \mathrm{~nm}$ was used to calculate the concentration of EEG in the product dispersion. An example spectrum is provided in Supplementary Fig. 20.

Annealing process: the annealing of CK-d powder was done in a tube furnace (Thermo Scientific Lindberg/Blue $M$ ) under an argon atmosphere. During annealing, the sample was heated at the rate of $10^{\circ} \mathrm{C} / \mathrm{min}$. CK-1d was annealed at $500^{\circ} \mathrm{C}$ for $12 \mathrm{~h}$ and $24 \mathrm{~h}$ and at $900^{\circ} \mathrm{C}$ and $1100^{\circ} \mathrm{C}$ for $12 \mathrm{~h}$. The annealed samples were designated as CK-1d A1, CK-1d A2, CK-1d $A 3$, and CK-1d A4, respectively. After cooling, the powder sample was collected and dispersed in water by bath sonication. Buckypaper of the annealed sample was prepared through a vacuum filtration process.

\section{Characterization techniques}

Imaging of the parent coke materials was done on cold emission SEM Hitachi S-4300 at an accelerating voltage of $15 \mathrm{keV}$ using a secondary electron detector. Pieces of coke were directly deposited on the SEM sample holder with adhesive carbon film. The coke was conductive enough that no beam charging was observed.

Raman spectra were measured using Horiba Jobin-Yvon LabRam HR with laser wavelength of $633 \mathrm{~nm}$, laser power of $1.91 \mathrm{~mW}$, exposure time of $10 \mathrm{~s}$, and three accumulations. The "Auto" baseline correction method in LabSpec was used for baseline removal.

TGA and derivative thermogravimetric (DTG) traces were recorded using a TA instrument. For recording the TG/DTG traces, a heating rate of $10^{\circ} \mathrm{C} /$ min and a sample size of $3 \pm 1 \mathrm{mg}$ were used under an $\mathrm{N}_{2}$ atmosphere (flow rate $60 \mathrm{~mL} / \mathrm{min}$ ).

Transmission electron microscopy was performed on a JEOL F200 cold emission transmission electron microscope. Coke was ground for imaging and suspended in alcohol. Exfoliated material was also suspended in alcohol. The suspensions were sonicated and a droplet of the solution was deposited on a TEM grid with lacey carbon support film. Imaging was done at room temperature using $200 \mathrm{keV}$ acceleration voltage. TEM images and diffraction patterns were recorded with in situ One View camera (Gatan, Inc.). Both coke and exfoliated material appeared stable under irradiation even at high doses required for high-resolution imaging.

Energy loss spectroscopy was performed with a JEOL F200 cold emission transmission electron microscope operated in scanning transmission electron microscopy mode. An annular dark field detector was used to search the sample and identify the appropriate spot for data collection. The spectrum image was recorded from a uniformly flat part of the particle. Data collection was done with a combination of a Digiscan device (Gatan Inc), and a Quantum GIF (Gatan Inc) operated in spectroscopy mode. Later, individual spectra from the spectrum image were summed to reduce the noise associated with low dose data collection.

The surface chemistry of CK-1d was probed using an Omicron X-ray photoelectron spectrometer employing an Mg-sourced X-ray beam to irradiate the sample surface. The emitted photoelectrons from the sample surface were collected by a $180^{\circ}$ hemispherical electron energy analyzer. A takeoff angle of $40^{\circ}$ between the sample surface and the path to the photoelectron collector was used in all measurements. During all scanning, charge neutralization by a dual-beam charge neutralizer was performed to irradiate the low-energy electrons to eliminate the binding energy shifts in the recorded spectra. The sample was dried under vacuum for $24 \mathrm{~h}$ to prevent any outgassing. High-resolution spectra were recorded at pass energy (constant analyzer energy) of $30.0 \mathrm{eV}$ with a step size of $0.05 \mathrm{eV}$. Component peak fitting and quantification of the spectra were carried out using CasaXPS curve fitting software (version 2.3.16).

Electrical conductivity was measured on buckypapers produced by vacuum filtration (Fisher Scientific MaximaDry) using a four-point resistivity probe powered by Keithley 2000, 6221, and two 6514 .

\section{DATA AVAILABILITY}

The data sets generated during and analyzed during the current study are available from the corresponding author upon reasonable request.
Received: 9 April 2021; Accepted: 5 August 2021; Published online: 23 August 2021

\section{REFERENCES}

1. Andrews, A. \& Lattanzio, R. K. Petroleum coke: Industry and environmental issues. (Congressional Research Service, 2013).

2. Shan, Y. et al. Rapid growth of petroleum coke consumption and its related emissions in China. Appl. Energy 226, 494-502 (2018).

3. Liang, M., Luo, B. \& Zhi, L. Application of graphene and graphene-based materials in clean energy-related devices. Int. J. Energy Res. 33, 1161-1170 (2009).

4. Jo, G. et al. The application of graphene as electrodes in electrical and optical devices. Nanotechnology 23, 112001 (2012).

5. Survey, U. G., S, O. \& Survey, U. G. Mineral Commodity Summaries. (Government Printing Office, 2009).

6. Needle Coke Market: Global Industry, Size, Share, Growth, Trends, and Forecast, 2018-2026. Transparency Market Research (2018).

7. Li, L. et al. Characteristics of the mesophase and needle coke derived from the blended coal tar and biomass tar pitch. J. Anal. Appl. Pyrolysis 150, 104889 (2020).

8. Ko, S., Kwon, Y. J., Lee, J. U. \& Jeon, Y.-P. Preparation of synthetic graphite from waste PET plastic. J. Ind. Eng. Chem. 83, 449-458 (2020).

9. Ren, S., Rong, P. \& Yu, Q. Preparations, properties and applications of graphene in functional devices: a concise review. Ceram. Int. 44, 11940-11955 (2018).

10. Sierra, U. et al. Cokes of different origin as precursors of graphene oxide. Fuel 166, 400-403 (2016)

11. Botas, C. et al. The effect of the parent graphite on the structure of graphene oxide. Carbon 50, 275-282 (2012).

12. Sierra, U. et al. New alternatives to graphite for producing graphene materials. Carbon 93, 812-818 (2015)

13. Xing, X., Zhang, X., Zhang, K., Jin, L. E. \& Cao, Q. Preparation of large-sized graphene from needle coke and the adsorption for malachite green with its graphene oxide. Fuller. Nanotubes Carbon Nanostruct. 27, 97-105 (2019).

14. Sierra, U. et al. Coke-derived few layer graphene-like materials by mild planetary milling exfoliation. Fuel 262, 116455 (2020).

15. Achee, T. C. et al. High-yield scalable graphene nanosheet production from compressed graphite using electrochemical exfoliation. Sci. Rep. 8, 14525 (2018).

16. Hope, J. T. et al. Scalable production of graphene nanoplatelets for energy storage. ACS Appl. Nano Mater., https://doi.org/10.1021/acsanm.0c02209 (2020).

17. $\mathrm{He}, \mathrm{M}$. et al. Mass production of tunable multicolor graphene quantum dots from an energy resource of coke by a one-step electrochemical exfoliation. Carbon 140, 508-520 (2018)

18. Zhamu, A. \& Jang, B. Z., Porous graphene/carbon composite balls for an alkal metal battery anode (Google Patents, 2020)

19. Rao, R. et al. Carbon nanotubes and related nanomaterials: critical advances and challenges for synthesis toward mainstream commercial applications. ACS Nano 12, 11756-11784 (2018).

20. Awadallah, A. E., Abdel-Hamid, S. M., El-Desouki, D. S., Aboul-Enein, A. A. \& AboulGheit, A. K. Synthesis of carbon nanotubes by CCVD of natural gas using hydrotreating catalysts. Egypt. J. Pet. 21, 101-107 (2012).

21. Ibrahimov, H. et al. Carbon nanotubes obtained from natural gas by CVD. J. Surf. Investig.: X-ray Synchrotron Neutron Tech. 13, 1244-1247 (2019).

22. Wang, I.-W., Kutteri, D. A., Gao, B., Tian, H. \& Hu, J. Methane pyrolysis for carbon nanotubes and $\mathrm{CO}$ x-free $\mathrm{H} 2$ over transition-metal catalysts. Energy Fuels 33, 197-205 (2018).

23. Mordkovich, V. et al. Synthesis of carbon nanotubes by catalytic conversion of methane: competition between active components of catalyst. Carbon 45, 62-69 (2007).

24. Rountree, K. S., Shah, S. A., Sweeney, C. B., Irin, F. \& Green, M. J. Graphene reflux: improving the yield of liquid-exfoliated nanosheets through repeated separation techniques. Nanotechnology 27, 505601 (2016).

25. Al-Gaashani, R., Najjar, A., Zakaria, Y., Mansour, S. \& Atieh, M. A. XPS and structural studies of high quality graphene oxide and reduced graphene oxide prepared by different chemical oxidation methods. Ceram. Int. 45, 14439-14448 (2019).

26. Tararan, A., Zobelli, A., Benito, A. M., Maser, W. K. \& Stéphan, O. Revisiting graphene oxide chemistry via spatially-resolved electron energy loss spectroscopy. Chem. Mater. 28, 3741-3748 (2016)

27. Park, M., Zhang, X., Chung, M., Less, G. B. \& Sastry, A. M. A review of conduction phenomena in Li-ion batteries. J. Power Sources 195, 7904-7929 (2010).

28. Ashuri, M., He, Q. \& Shaw, L. L. Silicon as a potential anode material for Li-ion batteries: where size, geometry and structure matter. Nanoscale 8, 74-103 (2016)

29. Mochida, I., Fujimoto, K.-i. \& Oyama, T. Chemistry in the production and utilization of needle coke. Vol. 24 (Marcel Dekker, Inc: New York-Basel, 1994). 
30. Franklin, R. E. Crystallite growth in graphitizing and non-graphitizing carbons. Proc. R. Soc. Lond. Ser. A. Math. 209, 196-218 (1951).

31. Sawarkar, A. N., Pandit, A. B. \& Samant, S. D. Petroleum residue upgrading via delayed coking: a review. Can. J. Chem. Eng. 85, 1-24 (2007).

32. Mancuso, L. \& Arienti, S. in Integrated Gasification Combined Cycle (IGCC) Technologies. 121-144 (Elsevier, 2017).

33. Wajid, A. S. et al. Polymer-stabilized graphene dispersions at high concentrations in organic solvents for composite production. Carbon 50, 526-534 (2012).

34. Wang, C. W., Yi, Y. B., Sastry, A. M., Shim, J. \& Striebel, K. A. Particle compression and conductivity in Li-ion anodes with graphite additives. J. Electrochem. Soc. 151, A1489 (2004).

\section{ACKNOWLEDGEMENTS}

We acknowledge the TAMU Materials Characterization Facility for the use of SEM facilities. We appreciate Julie $\mathrm{Oh}$ and Huaixuan Cao of TAMU for help in characterization. Funding was provided by the ExxonMobil Chemical Company.

\section{AUTHOR CONTRIBUTIONS}

Experimental work: S.S., P.L., M.J.M., B.J.C., K.A., S.Y., X.Z. Experimental design and analysis: S.S., P.L., S.Y., S.U., M.J.G., R.A.H. Writing the paper: S.S., P.L., M.J.M., B.J.C., S.Y., S.U., M.J.G., R.A.H.

\section{COMPETING INTERESTS}

The findings described in this paper are closely related to intellectual property held by TAMU and ExxonMobil Chemical Company.

\section{ADDITIONAL INFORMATION}

Supplementary information The online version contains supplementary material available at https://doi.org/10.1038/s41699-021-00255-8.

Correspondence and requests for materials should be addressed to M.J.G. or R.A.H.

Reprints and permission information is available at http://www.nature.com/ reprints

Publisher's note Springer Nature remains neutral with regard to jurisdictional claims in published maps and institutional affiliations.

\section{(c) (i)}

Open Access This article is licensed under a Creative Commons Attribution 4.0 International License, which permits use, sharing, adaptation, distribution and reproduction in any medium or format, as long as you give appropriate credit to the original author(s) and the source, provide a link to the Creative Commons license, and indicate if changes were made. The images or other third party material in this article are included in the article's Creative Commons license, unless indicated otherwise in a credit line to the material. If material is not included in the article's Creative Commons license and your intended use is not permitted by statutory regulation or exceeds the permitted use, you will need to obtain permission directly from the copyright holder. To view a copy of this license, visit http://creativecommons. org/licenses/by/4.0/.

(c) The Author(s) 2021, corrected publication 2021 